\title{
Cyclodextrins: A Potential Tool for Studying the Role of Glycerolipids in Photosynthetic Membranes
}

\author{
Sylvie Duchêne and Paul-André Siegenthaler* \\ Laboratoire de Physiologie végétale, Université de Neuchâtel, CH-2007 Neuchâtel, Switzerland
}

\begin{abstract}
A novel cyclodextrin derivative, i.e., permethylated- $\alpha$-cyclodextrin (PM- $\alpha-C D)$, was used for removing glycerolipids from spinach thylakoid membranes and investigating their role in photosynthetic activities. A three-step selective removal of each lipid class was observed in treated membranes. Up to a concentration of $4 \mathrm{mM}, \mathrm{PM}-\alpha-\mathrm{CD}$ (in the presence of $75 \mu \mathrm{g}$ chlorophyll $a+b / \mathrm{mL}), \mathrm{PM}-\alpha-\mathrm{CD}$ displayed a marked selectivity for anionic lipids [sulfoquinovosyldiacylglycerol (SQDG) and phosphatidylglycerol (PG)] in comparison with galactolipids. At this concentration, half of PG and SQDG were removed. Within this range of concentration, the volume response of treated thylakoids to variation of osmolarity, an indirect mean of verifying the structural integrity of the membrane, was not altered. Similarly, neither photosystem II (PSII) nor photosystem I (PSI) activity was affected. In contrast, the low-temperature fluorescence ratio F695/F740 drastically diminished from 1.45 to about 0.7, essentially due to the decrease of PSII fluorescence. The results derived from the fast fluorescence rise expressed in the form of a spider suggest that the fraction of inactivated (non- $\mathrm{Q}_{\mathrm{A}}$ reducing) reaction centers $(\mathrm{RC})$ increases while the active $\left(Q_{A}\right.$ reducing) $R C$ remained intact. Raising the concentration of PM- $\alpha$-CD from 4 to $7 \mathrm{mM}$ resulted in a progressive but greater diminution of the galactolipid level than that of SQDG and PG. Within this concentration range, the integrity of the membrane was not altered, nor was either PSII or PSI activity, whereas the F695/F740 ratio decreased to about 0.45 as well as the fraction of inactivated RC. At concentrations above $7 \mathrm{mM}$ of PM- $\alpha-C D$, the integrity of the membrane was impaired, resulting in a decrease of both electron transport activities. At all concentrations, PM- $\alpha-C D$ did not show any selectivity toward either the acyl chains of the lipid molecules or the molecular species of PG. The results are discussed in terms of the role of glycerolipids in thylakoid membrane function and the relationship of the chemical structure of PM- $\alpha-C D$ and its lipid removal capacity.

Paper no. L8865 in Lipids 37, 201-208 (February 2002).
\end{abstract}

\footnotetext{
*To whom correspondence should be addressed at Laboratoire de Physiologie végétale, Université de Neuchâtel, Rue Emile-Argand 13, CH-2007 Neuchâtel, Switzerland.

E-mail: Paul-Andre.Siegenthaler@unine.ch

Abbreviations: ABS, light absorption; $\mathrm{CD}$, cyclodextrin; $\alpha$-CD, $\alpha$-cyclodextrin; $\beta$-CD, $\beta$-cyclodextrin; $\gamma$-CD, $\gamma$-cyclodextrin; Chl, chlorophyll; CSo, foliar cross section; DGDG, digalactosyldiacylglycerol; $2,6-\mathrm{DM}-\beta-\mathrm{CD}, 2,6-$ dimethyl- $\beta$-cyclodextrin; ETo, photosystem II electron transfer; $\mathrm{F}_{695}$, fluorescence at $695 \mathrm{~nm} ; \mathrm{F}_{740}$, fluorescence at $740 \mathrm{~nm}$; LHCII, light harvesting chlorophyll $a / b$ protein complex; MGDG, monogalactosyldiacylglycerol; PG, phosphatidylglycerol; PLA $_{2}$, phospholipase $\mathrm{A}_{2} ; \mathrm{PM}-\alpha-\mathrm{CD}$, permethylated- $\alpha$-cyclodextrin; PSI, photosystem I; PSII, photosystem II; RC, active reaction center; SQDG, sulfoquinovosyldiacylglycerol; TM, thylakoid membranes; TRo, photon trapping.
}

Besides proteins, acyl lipids are major components of thylakoid membranes (TM) in higher plants. They consist of four classes: monogalactosyldiacylglycerol (MGDG), digalactosyldiacylglycerol (DGDG), sulfoquinovosyldiacylglycerol (SQDG), and phosphatidylglycerol (PG). Galactolipids (MGDG and DGDG) are prominent and account for about $80 \%$ of total glycerolipids. Moreover, they are characterized by an exceptionally high content of trienoic acid, mainly $\alpha$ linolenic acid (encountered in all lipid classes) and, in the socalled 16:3 plants, such as spinach, hexadecatrienoic acid (found in MGDG only). SQDG is enriched in palmitic acid (35 mol\%), whereas PG contains a unique FA, trans- $\Delta^{3}$-hexadecenoic acid $[16: 1(3 t)](1,2)$. The FA composition of these acyl lipids gives rise to a great number of molecular species $(3,4)$.

Glycerolipids display a marked transmembrane distribution in the TM. The outer monolayer is highly enriched in MGDG $(5,6)$ and PG $(7,8)$, whereas the inner one contains a high level of DGDG $(5,6)$; this confirms the general sidedness of TM components. In contrast, recent analyses of glycerolipids in different domains of the membrane (e.g., intact thylakoids, grana lamellae vesicles, central core of the appressed region, grana margin vesicles, and stroma lamellae vesicles) reveal that the level of the four lipid classes, the nature of their acyl chains, and the main molecular species of PG are identical in the intact TM as well as in the four membrane domains (9).

The role of membrane lipids in the photosynthetic function have been particularly difficult to study. Although several approaches have been proposed in the literature (for a review, see Ref. 10), there is no consensus about the results obtained. Among them, the enzymatic digestion of lipids (e.g., $5,6,8,11,12$ ), the use of specific antibodies against lipids (e.g., Refs. 13-15), the preparation of subthylakoid fractions $(16,17)$, and the use of mutants deficient in certain lipids (18) have been extensively studied. All these techniques have advantages and drawbacks. For instance, the use of enzymatic digestion with lipolytic enzymes results in the concomitant accumulation of FFA that are known to have detergentlike properties and to displace lipids in the membrane $(19,20)$. The use of specific antibodies against lipids is often questionable due to the difficult access of the antibody to all the lipids at the surface of the membrane, especially if they are located in membrane hollows. Although the preparation of subthylakoid fractions seemed to have been for a long time the best technique to associate structure and function, its greatest drawback comes from utilization of high concentrations of deter- 
gents. This explains why a few authors have tried to obtain subthylakoid particles or domains without detergent by using the aqueous two-phase system $(9,19,21,22)$. Although the mutant approach has permitted study of the effect of the degree of lipid polyunsaturation on the structure and function of the chloroplast membrane $(10,18)$, it is extremely difficult to evaluate the side effects of specific mutations on lipid metabolism.

Recently, cyclodextrins (CD) were reported to have the capacity to remove lipids from the TM (23) and display hemolytic properties (24). CD molecules are cyclic oligosaccharides consisting of $6(\alpha-C D), 7(\beta-C D)$, or $8(\gamma-C D)$ glucopyranose units linked by $\alpha(1-4)$ bonds. These compounds are not perfectly cylindrical molecules but rather are somewhat coneshaped. They adopt a torus shape and are able to bind lipids within their hydrophobic cavity to form water-soluble guestCD inclusion complexes (25-27). So far, CD have been used mainly in the biomedical and pharmaceutical fields.

In a previous contribution, we established the conditions under which TM glycerolipids can be extracted in the presence of $\alpha-C D, \beta-C D$, and dimethyl- $\beta$-cyclodextrin (DM- $\beta-C D)$ (23). We found that the extent of lipid, protein, pigment, and plastoquinone removal depends on both $\mathrm{CD}$ and chlorophyll (Chl) concentrations. Simultaneous with the removal of lipids, a few photosynthetic functions were found to be altered.

In this investigation, we used a novel $\mathrm{CD}$ derivative, the permethylated- $\alpha$-cyclodextrin (PM- $\alpha-C D)$. This compound was found to display properties different from those of the $\mathrm{CD}$ used previously. At low concentrations, it had the capacity of removing anionic lipids (SQDG and PG) without concomitant galactolipid extraction. These properties allowed us to study the relationship between the level of anionic lipids and various photosynthetic functions. Preliminary results of this investigation were presented elsewhere $(28,29)$.

\section{EXPERIMENTAL PROCEDURES}

Materials. All the CD used in this study were obtained from Fluka (Buchs, Switzerland). The PM- $\alpha-C D$ was made by Dr. S. Claude (Department of Organic Chemistry, University of Neuchâtel, Switzerland). All chemicals were high-purity products from Fluka or Sigma, and Percoll was provided by Pharmacia (Uppsala, Sweden). Spinach leaves were purchased from the local market. They were washed, stored at $4^{\circ} \mathrm{C}$ and used within $2 \mathrm{~d}$.

Preparation of TM. Spinach leaves $(150 \mathrm{~g})$ were ground for $10 \mathrm{~s}$ in $375 \mathrm{~mL}$ of a grinding medium containing $330 \mathrm{mM}$ sorbitol, $30 \mathrm{mM}$ MOPS-KOH (pH 7.8), 2 mM EDTA-Na 2 , and $0.15 \%$ BSA by using a Waring Blender. The mixture was filtered through four layers of cheesecloth and two layers of miracloth, and the filtrate was subsequently centrifuged at $1500 \times g$ for $2 \mathrm{~min}$. The supernatant was discarded by aspiration, and the intact chloroplast pellet was resuspended in the grinding medium $(20 \mathrm{~mL})$ and layered on two centrifuge swingingbucket tubes containing $20 \mathrm{~mL}$ of Percoll $40 \%$ supplemented with the grinding medium without EDTA- $\mathrm{Na}_{2}$ and BSA. After centrifugation $(2500 \times g, 10 \mathrm{~min})$, the chloroplast pellet was resuspended in a low-osmotic medium $(36 \mathrm{~mL})$ containing 10 $\mathrm{mM}$ Tricine-KOH ( $\mathrm{pH} 7.8$ ) and 2 mM EDTA- $\mathrm{Na}_{2}$ for $2 \mathrm{~min}$. The osmolarity was reajusted by adding back $4 \mathrm{~mL}$ of $3 \mathrm{M}$ sorbitol. After centrifugation at $13,400 \times g$ for $5 \mathrm{~min}$, the thylakoid pellets were resuspended in a washing medium containing 300 $\mathrm{mM}$ sorbitol and $10 \mathrm{mM}$ Tricine- $\mathrm{KOH}$ (pH 7.8). Fifteen milliliters of Percoll (5\% Percoll in $300 \mathrm{mM}$ sorbitol and $10 \mathrm{mM}$ Tricine-KOH pH 7.8) were injected slowly below the suspension $(20 \mathrm{~mL})$ of thylakoids. The tubes were centrifuged at $20,000 \times g$ for $10 \mathrm{~min}$, and the thylakoid pellets were washed in the same medium at $13,500 \times g$ for $5 \mathrm{~min}$ and then resuspended in the same medium at a concentration of $2 \mathrm{mg}$ $\mathrm{Chl} / \mathrm{mL}$.

Incubation of thylakoids in the presence of $C D$. All CD were solubilized in $300 \mathrm{mM}$ sorbitol and $10 \mathrm{mM}$ Tricine$\mathrm{KOH}(\mathrm{pH}$ 7.8). The concentration of the stock solutions was $10 \mathrm{mM}$. CD at the desired concentrations were added to the thylakoid preparation $(50-75 \mu \mathrm{g} \mathrm{Chl} / \mathrm{mL})$ and incubated at $0^{\circ} \mathrm{C}$ for $20-30 \mathrm{~min}$. At the end of the reaction, thylakoids were centrifuged at $14,500 \times g$ for $10 \mathrm{~min}$ and resuspended in $300 \mathrm{mM}$ sorbitol and $10 \mathrm{mM}$ Tricine- $\mathrm{KOH}$ (pH 7.8). The concentration of $\mathrm{Chl}$ was readjusted to 1 or $2 \mathrm{mg} \mathrm{Chl} / \mathrm{mL}$ depending on the fate of the preparation.

Lipid analyses. Total lipids were extracted by adding $4 \mathrm{~mL}$ chloroform/methanol (53:37, vol/vol) and $2 \mathrm{~mL} 0.5 \mathrm{M} \mathrm{KCl}$ to the thylakoid suspension $(150 \mu \mathrm{L}$ of thylakoids containing 1 $\mathrm{mg} \mathrm{Chl} / \mathrm{mL}$ ). This resulted in a two-phase system. The lipids of the lower phase were separated by TLC with acetone/ toluene/water (91:30:8) as solvents (30). Each lipid class was then hydrolyzed and esterified with $5 \% \mathrm{H}_{2} \mathrm{SO}_{4}$ in methanol at $85^{\circ} \mathrm{C}$ for $1 \mathrm{~h}$. The resulting FAME were separated and identified by GC and the molecular species of PG determined according to Xu and Siegenthaler (4). Chl concentrations were determined as described by Bruinsma (31).

Photosynthetic activities. Uncoupled photosystem II (PSII) electron flow was measured polarographically at $20^{\circ} \mathrm{C}$ in the presence of red actinic light $\left(29 \mathrm{~mW} / \mathrm{cm}^{2}\right)$ in a medium containing $30 \mathrm{mM}$ sodium phosphate buffer ( $\mathrm{pH} 6.5$ ), $3 \mathrm{mM}$ $\mathrm{NaCl}, 60 \mathrm{mM}$ sucrose, $0.2 \mathrm{mM}$ phenyl-p-benzoquinone, 2 $\mathrm{mM} \mathrm{NH}{ }_{4} \mathrm{Cl}$, and thylakoids corresponding to $20 \mu \mathrm{g} \mathrm{Chl} / \mathrm{mL}$, using a Clark-type oxygen electrode (evolution of $\mathrm{O}_{2}$ ). Uncoupled photosystem I (PSI) electron flow was determined in a medium containing $40 \mathrm{mM}$ sodium phosphate buffer $(\mathrm{pH}$ 7.4), $1 \mathrm{mM} \mathrm{NaCl}, 10 \mu \mathrm{M}$ 3-(3,4-dichlorophenyl)-1,1-dimethylurea; $32 \mathrm{mM}$ ascorbate, $0.3 \mathrm{mM}, 0.12 \mathrm{mM}$ methylviologen, $0.6 \mathrm{mM} \mathrm{NaN}_{3}, 2 \mathrm{mM} \mathrm{NH}_{4} \mathrm{Cl}$ and thylakoids corresponding to $20 \mu \mathrm{g} \mathrm{Chl} / \mathrm{mL}$, using a Clark-type electrode (absorption of $\mathrm{O}_{2}$ ) (8). Fluoresence studies were carried out with freshly prepared thylakoids resuspended in $300 \mathrm{mM}$ sorbitol and $10 \mathrm{mM}$ Tricine- $\mathrm{KOH}$ (pH 7.8). The low-temperature fluorescence spectra of thylakoids (diluted 1:9 in $80 \%$ glycerol) were carried out according to Rawyler and Siegenthaler (23). The fluorescence transients OJIP were measured as described by Srivastava and Strasser (32).

Response of thylakoids to osmolarity variation. The integrity of thylakoid membranes during the removal of lipids 
by $\mathrm{CD}$ was estimated by an osmolarity test $(7,33)$. Thylakoids were treated with various concentrations of PM- $\alpha-C D(4,6$, and $9.6 \mathrm{mM})$ as indicated previously. Then, each sample (2 $\mathrm{mg} \mathrm{Chl} / \mathrm{mL}$ ), including the control without $\mathrm{CD}$, was suspended in $40 \mathrm{mM}$ Tricine-KOH ( $\mathrm{pH} 8.0$ ) and $3 \mathrm{mM} \mathrm{MgCl}_{2}$ and divided into four equal parts. Increasing concentrations of sucrose $(40,51$, and $60 \mathrm{mM})$ were then added to each of the samples. Osmotic equilibrium was reached after $5 \mathrm{~min}$, and then the thylakoid preparations were transferred to hematocrit tubes and centrifuged at 23,000 $\times g$ (microcentrifuge; Christ, Zurich, Switzerland) for $5 \mathrm{~min}$. The height of the thylakoid sediment in the tube was measured and compared to the total volume of the original suspension (ratio packed volume/total volume, arbitrary units).

\section{RESULTS}

Several CD ( $\alpha-, \beta-, \gamma-C D)$ and $C D$ derivatives were compared regarding their lipid removal capacity and selectivity. The results are presented in Table 1. $\alpha$-CD [which contains six glycopyranosidic units and presents an internal diameter of the cavity of $5.2 \AA$ (27)] and its permethylated- $\alpha$-derivative (PM$\alpha-C D)$ displayed a much greater preference for SQDG and PG than for galactolipids. In contrast, $\beta$-CD [which contains seven glycopyranosidic units and presents an internal diameter of the cavity of $6.4 \AA(27)$ ] removed roughly $11 \mathrm{~mol} \%$ of the four lipid classes. The derivative of $\beta-\mathrm{CD}(2,6-\mathrm{DM}-\beta-\mathrm{CD})$ displayed a preference for the anionic lipids (SQDG and PG) over galactolipids but to a lesser extent than the $\alpha-C D$ and PM- $\alpha$-CD. Other $\beta$-derivatives, such as permethylated- $\beta$-, carboxylated- $\beta-$, quaternary- $\beta-$, amphiphilic- $\beta$-, and sulfated- $\beta-C D$, removed only lipids in trace amounts (data not shown). The selective capacity of $\gamma$-CD [which contains eight glycopyranosidic units and presents an internal diameter of the cavity of $8.3 \AA$ (27)] was greater for SQDG than for all other lipid classes. But the amount of SQDG removed was rather low. A mixture of $\alpha$ - and $\beta$-CD removed about 30 mol\% of galactolipids and $60 \mathrm{~mol} \%$ of SQDG and PG but had no selective effect for one particular lipid class (Table 1). On the basis of these results, we focused our attention on the effect of PM- $\alpha-C D$.

The results in Figure 1 show that the removability of total lipids in the thylakoid membrane increased as a function of the concentration of PM- $\alpha-C D$ up to $8 \mathrm{mM}$. At this concentration, $50 \mathrm{~mol} \%$ of the total lipids were removed. A further addition of PM- $\alpha-C D$ had no effect.

Figure 2 illustrates the selective removal of each lipid class as a function of PM- $\alpha-C D$ concentration. Three steps can be distinguished: (i) Up to a concentration of $4 \mathrm{mM}$ PM- $\alpha-C D$, the content in MGDG remained constant, while that of DGDG decreased slightly. In contrast, the amount in anionic lipids decreased approximately by half of its original level. (ii) Raising the concentration of PM- $\alpha-C D$ from 4 to $8 \mathrm{mM}$ resulted in a progressive but greater diminution of the galactolipid level than that of SQDG and PG. (iii) Higher concentrations of PM- $\alpha-C D$ did not affect significantly the content of all four lipid classes. The concentration of PM- $\alpha-C D$ necessary to remove $50 \mathrm{~mol} \%$ of each lipid was $8,9.6,3$, and 3.5 $\mathrm{mM}$ for MGDG, DGDG, SQDG, and PG, respectively (see dotted lines in Fig. 2).

Figure 3 shows the relative content and composition of fatty acids in each lipid class remaining in the thylakoid membrane after a treatment with various concentrations of PM- $\alpha$ CD. The PM- $\alpha-C D$ did not show any selectivity toward the acyl chains of the lipid molecules.

Spinach thylakoid membranes contain 10 PG molecular species (4). The three main molecular species [18:3/16:1 (3t); $18: 3 / 16: 0 ; 16: 0 / 16: 1(3 t)]$ represented $94.9 \mathrm{~mol} \%$ of the total molecular species. The effect of PM- $\alpha-C D(0,2,5$, and 9.6 $\mathrm{mM}$ ) on their content in treated thylakoids was found to be 79.5 to $82.3 \mathrm{~mol} \%$ for $18: 3 / 16: 1(3 t), 6.4$ to $8.0 \mathrm{~mol} \%$ for 18:3/16:0, and 3.2 to 5.6 mol\% for $16: 0 / 16: 1(3 t)$. All these values presented no statistical difference between the control values and those of the different concentrations of PM- $\alpha-C D$ (individual data not shown). In conclusion, PM- $\alpha-C D$ did not show any selectivity toward the molecular species of PG, i.e., each molecular species was removed in the same proportion at each concentration of the $\mathrm{CD}$.

TABLE 1

Comparison of the Removability of Lipid Classes in Thylakoids Treated by Various Cyclodextrins (CD)

\begin{tabular}{|c|c|c|c|c|c|}
\hline \multirow[b]{2}{*}{ CD used ${ }^{a}$} & \multicolumn{4}{|c|}{ Lipid removal (mol\%) } & \multirow[b]{2}{*}{$n$} \\
\hline & MGDG & DGDG & SQDG & PG & \\
\hline$\alpha-\mathrm{CD}^{b}$ & $5.4 \pm 4.8$ & $5.4 \pm 2.7$ & $42.4 \pm 2.1$ & $39.1 \pm 7.4$ & 4 \\
\hline$P M-\alpha-C D^{c}$ & $12 \pm 4$ & $15 \pm 2$ & $64 \pm 5$ & $54 \pm 5$ & 6 \\
\hline$\beta-C D^{b}$ & $13.9 \pm 2.4$ & $14.5 \pm 3$ & $8.1 \pm 3.5$ & $12.5 \pm 2.6$ & 4 \\
\hline $2,6-\mathrm{DM}-\beta-C D^{b}$ & $28.1 \pm 3.5$ & $17.8 \pm 2.1$ & $43.7 \pm 6.4$ & $38.3 \pm 2.8$ & 4 \\
\hline$\gamma-\mathrm{CD}^{c}$ & $5 \pm 3$ & Trace & $22 \pm 7$ & $8 \pm 6$ & 3 \\
\hline$\alpha-+\beta-C D^{b}$ & $27.7 \pm 5.0$ & $27.0 \pm 5.5$ & $60.0 \pm 6.4$ & $54.4 \pm 5.9$ & 6 \\
\hline
\end{tabular}

Thylakoids were incubated with $5 \mathrm{mM}$ of one of the cyclodextrins in a medium containing $300 \mathrm{mM}$ sorbitol and $10 \mathrm{mM}$ Tricine- $\mathrm{KOH}\left(\mathrm{pH} \mathrm{7.8)}\right.$ ) at $0^{\circ} \mathrm{C}$.

$b_{50} \mu \mathrm{g}$ chlorophyll/mL; $30 \mathrm{~min}$ incubation [results from Rawyler and Siegenthaler (23)].

${ }^{c} 75 \mu \mathrm{g}$ chlorophyll/mL; 20 min incubation. MGDG, monogalactosyldiacylglycerol; DGDG, digalactosyldiacylglycerol; SQDG, sulfoquinovosyldiacylglycerol; PG, phosphatidylglycerol. 


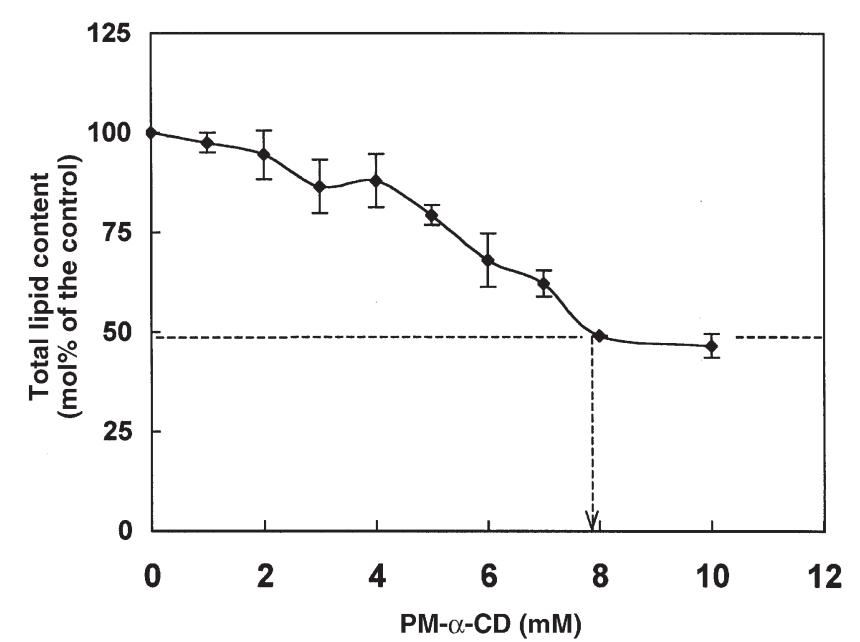

FIG. 1. Changes in the relative content of total lipids in thylakoid membranes treated with various concentrations of permethylated $\alpha$ cyclodextrin (PM- $\alpha-C D)$. The SD was calculated on the basis of six experiments $(n=6)$. The dotted line shows that $50 \mathrm{~mol} \%$ of the total lipids was removed in the presence of $8 \mathrm{mM}$ PM- $\alpha$-CD. The $100 \%$ value corresponded to $2600 \pm 100 \mathrm{nmol} / \mathrm{mg}$ chlorophyll (Chl).

The effect of PM- $\alpha-C D$ on electron transport activity in spinach thylakoids is shown in Table 2. Up to a concentration of $7 \mathrm{mM}$ PM- $\alpha-C D$, neither PSII nor PSI activity was affected. However, a small stimulation of PSI activity at $5 \mathrm{mM}$ PM- $\alpha-C D$ could be detected. At a concentration of $7 \mathrm{mM}, 30$ mol\% of each galactolipid, $70 \mathrm{~mol} \%$ of SQDG, and $60 \mathrm{~mol} \%$ of PG were removed by this treatment (Fig. 2). A further increase of PM- $\alpha-\mathrm{CD}$ (up to $9.6 \mathrm{mM}$ ) inhibited both activities,

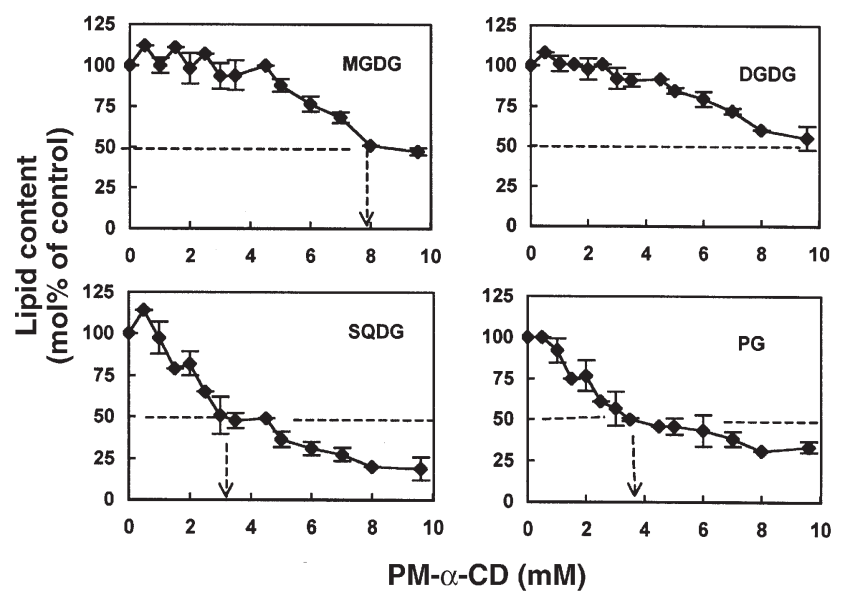

FIG. 2. Changes in the relative amount of individual diacyl lipid classes (MGDG, DGDG, SQDG, PG) in thylakoid membranes treated with PM$\alpha-C D$. The standard deviation was calculated on the basis of nine experiments $(n=9)$. The dotted lines indicate the concentration at which PM- $\alpha-C D$ was necessary to remove 50 mol\% of each lipid class. For each of the four lipid classes, the control amount, given in nmol lipid per mg Chl, was MGDG (1400), DGDG (707), SQDG (165), and PG (227). MGDG, monogalactosyldiacylglycerol; DGDG, digalactosyldiacylglycerol; SQDQ, sulfoquinovosyldiacylglycerol; PG, phosphatidylglycerol; for other abbreviation see Figure 1.
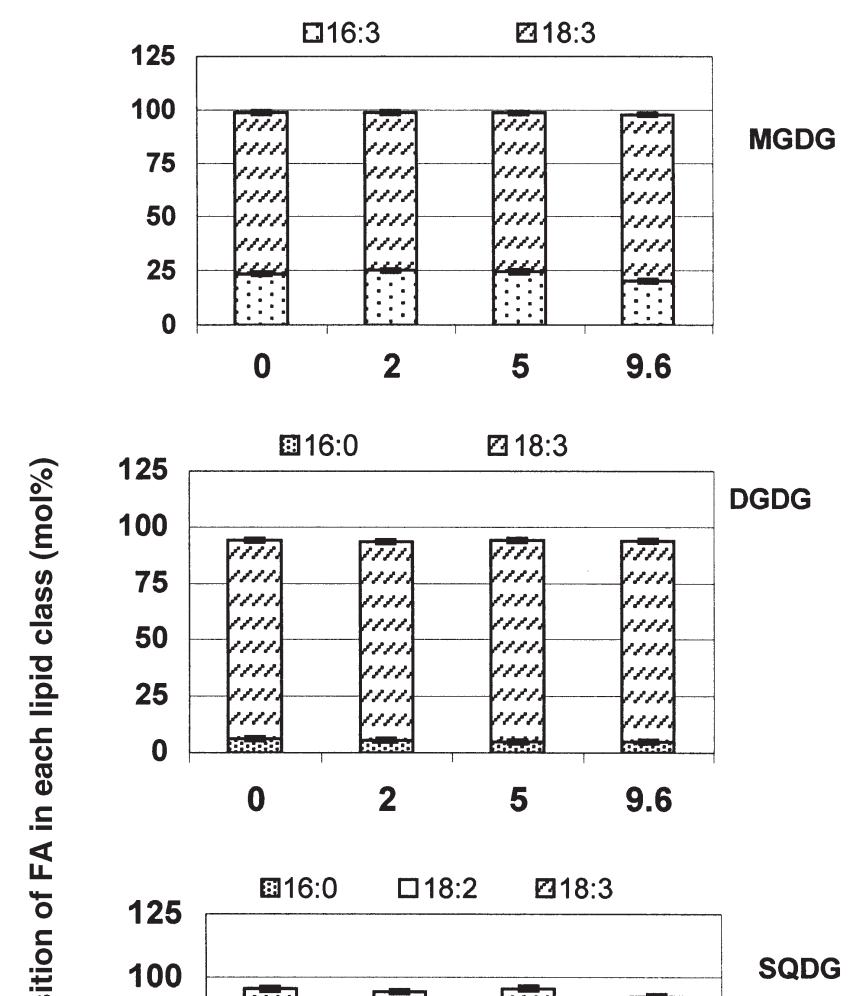

FIG. 3. Relative content and composition of FA in each lipid class remaining in the thylakoid membrane after treatment with PM- $\alpha-C D$. Only the main FA of each lipid class are illustrated in this graph. This explains why some $100 \%$ values are not reached (e.g., DGDG and SQDG). The standard deviation was calculated on the basis of seven experiments $(n=7)$. The $100 \%$ values of the controls ( 0 mM PM- $\alpha-C D)$ corresponded in nmol per $\mathrm{mg}$ Chl to 1400 for MGDG, 707 for DGDG, 165 for SQDG, and 227 for PG. The $100 \%$ values for all the other histograms are different and can be calculated from the results of Figure 2. See Figures 1 and 2 for abbreviations.

although to different extents for PSII (35\% inhibition) and PSI (56\% inhibition). At this concentration, an additional treatment of the membrane with phospholipase $\mathrm{A}_{2}\left(\mathrm{PLA}_{2}\right)$ 
TABLE 2

Effect of PM- $\alpha$-cyclodextrin on Photosystems II and I Electron Transport Activity in Spinach Thylakoids

\begin{tabular}{lcc}
\hline $\begin{array}{l}\text { PM- } \alpha-C D \\
(\mathrm{mM})\end{array}$ & $\begin{array}{c}\text { Photosystem II (PSII) } \\
\text { activity }(\%)^{a}\end{array}$ & $\begin{array}{c}\text { Photosystem I (PSI) } \\
\text { activity }(\%)^{b}\end{array}$ \\
\hline 0 & 100 & 100 \\
2 & $97 \pm 11(n=5)$ & $98 \pm 6(n=5)$ \\
5 & $95 \pm 14(n=5)$ & $132 \pm 24(n=5)$ \\
7 & $92 \pm 12(n=5)$ & $104 \pm 6(n=5)$ \\
9.6 & $65 \pm 11(n=6)$ & $44 \pm 13(n=7)$ \\
$9.6+\mathrm{PLA}_{2}{ }^{c}$ & $33 \pm 6(n=2)$ & $45 \pm 3(\mathrm{n}=2)$ \\
\hline
\end{tabular}

${ }^{a}$ PSII electron transport actitivy: $100 \%$ corresponded to $36 \pm 12$ micromol $\mathrm{O}_{2} / \mathrm{mg} \mathrm{Chl} \times \mathrm{h}(n=7)$.

${ }^{b}$ PSI electron transport activity: $100 \%$ corresponded to $460 \pm 90$ micromol $\mathrm{O}_{2} / \mathrm{mg} \mathrm{Chl} \times \mathrm{h}(n=7)$.

${ }^{C}{ }^{2} A_{2}$, phospholipase $A_{2}$ from porcine pancreas. After the permethylated- $\alpha-$ cyclodextrin (PM- $\alpha$-CD) treatment $\left(20 \mathrm{~min}, 2^{\circ} \mathrm{C}\right), \mathrm{PLA}_{2}$ (4 Boehringer units $/ \mathrm{mg} \mathrm{Chl}$ ) was added to the same reaction mixture and incubated at $20^{\circ} \mathrm{C}$ for $40 \mathrm{~min}$.

further decreased PSII activity from 65 to $33 \%$ but had no effect on PSI activity (Table 2).

It was important to verify the structural state of the membrane in PM- $\alpha-C D$-treated thylakoids. This was achieved by testing the volume response of thylakoids to variations of the osmolarity (33). As shown in Figure 4, an increase in the osmolarity (from 49 to $109 \mathrm{Osmol}$ ) resulted in a decrease of about half of the packed volume of thylakoids. This occurred not only in the control samples but also in thylakoids treated with 4 and $6 \mathrm{mM} \mathrm{PM- \alpha -CD}$. However, in the presence of 9.6 mM PM- $\alpha$-CD (Fig. 4) or 9.6 mM PM- $\alpha-C D+$ PLA $_{2}$ (data not shown), the osmotic response was nearly abolished.

Figure 5 shows the effect of various concentrations of PM$\alpha$-CD on low-temperature fluorescence. In the control thylakoid membrane, the ratio of fluorescence at $695 \mathrm{vs} .740 \mathrm{~nm}$ (F695/F740) was 1.45, indicating that the PSII fluorescence was greater than that of PSI. This ratio decreased progressively when the concentration of $\mathrm{CD}$ was raised. The extent

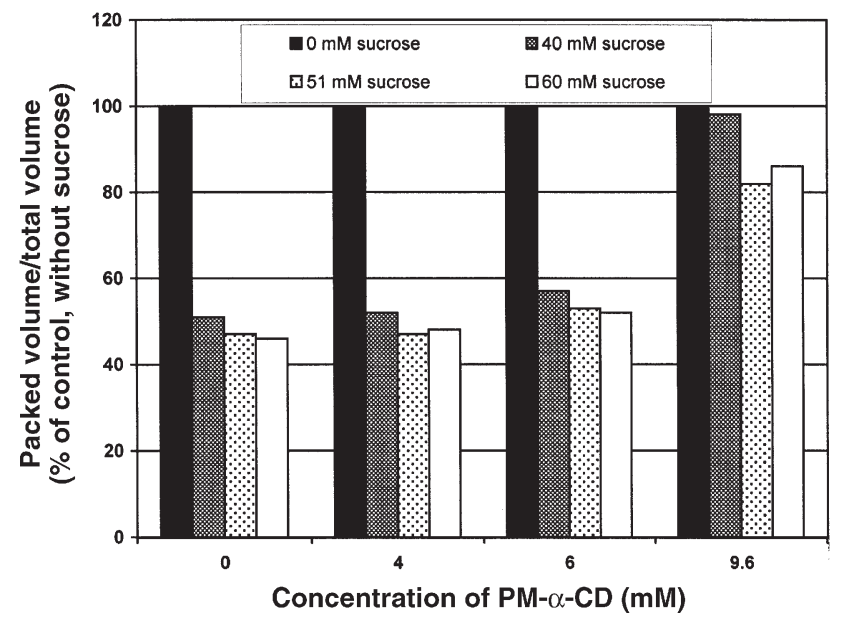

FIG. 4. Osmotic response of thylakoids first pretreated by 4, 6, and 9.6 $\mathrm{mM}$ PM- $\alpha-C D$, and then incubated in a medium containing $0,40,51$, or $60 \mathrm{mM}$ sucrose corresponding to 49, 89, 100, and 109 Osmol, respectively (other conditions as in the Experimental Procedure section). For abbreviation see Figure 1.

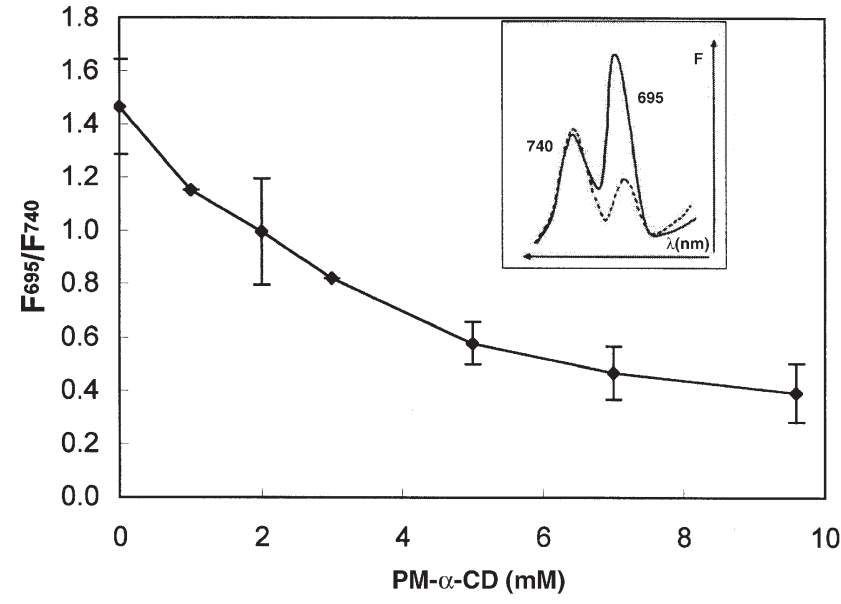

FIG. 5. Effect of lipid depletion by PM- $\alpha-C D$ on the low-temperature (77 K) fluorescence expressed as the ratio of the fluorescence at $695 \mathrm{~nm}$ (photosystem II: PSII) to the fluoresence at $740 \mathrm{~nm}$ (photosystem I: PSI) in thylakoid membranes. Where indicated, the SD was calculated on the basis of six experiments $(n=6)$. For the other ones, $n=2$. Inset: fluorescence spectra at $77 \mathrm{~K}$ of control sample (solid line) and PM- $\alpha$-CD-treated ( $5 \mathrm{mM}$ ) thylakoids (dashed line). The shift of wavelength at $695 \mathrm{~nm}$ between the two samples is not significant. For abbreviation see Figure 1.

of this decrease was greater at low than at high concentrations of PM- $\alpha-C D$. At a concentration of $4 \mathrm{mM}$, the ratio corresponded to half of the value of the original one. As shown in the inset of Figure 5, the decrease in the F695/F740 ratio was entirely due to the decrease of the fluorescence at $695 \mathrm{~nm}$ in the presence of PM- $\alpha-C D$.

The results derived from the fast fluorescence rise (OJIP) $(32,34)$ of CD-treated thylakoids are illustrated as a spiderplot presentation in Figure 6. This is a multiparametric description of structure and function of each photosynthetic

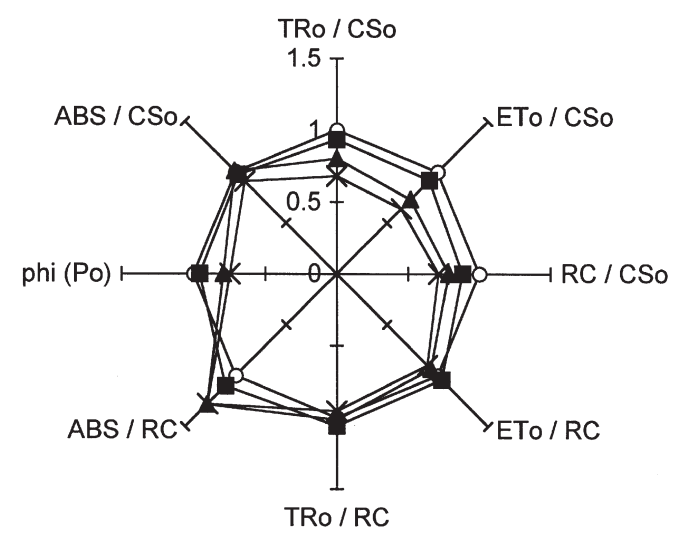

FIG. 6. Effect of lipid depletion by PM- $\alpha-C D$ on various parameters characterizing the photochemistry of the thylakoid membranes; ABS, light absorption; TRo, photon trapping; ETo, PSII electron transfer; RC, active reaction center; CSo, foliar cross section; phi $(\mathrm{Po})=\mathrm{TRo} / \mathrm{ABS}$, yield of primary photochemistry. Symbols: $\bigcirc$, $0 \mathrm{mM} \mathrm{PM-} \alpha-C D ; \boldsymbol{\square}, 2$ $\mathrm{mM} ; \boldsymbol{\Delta}, 4 \mathrm{mM} ; \mathrm{x}, 6 \mathrm{mM}$. The scale from 0 (center of the spider) to 1.5 corresponds to ratios of the various parameters of a treated sample and its control. For other abbreviation see Figure 1. 
sample (control and PM- $\alpha$-CD-treated thylakoids), presented by an octagonal line. This type of presentation facilitates the comparison of the effect of different treatments. The relative values (relative to the corresponding value of the control, which thus become equal to unity) of selected expressions, such as PSII electron transfer/cross-foliar section (ETo/CSo) and $\mathrm{ETo} /$ active reaction center $(\mathrm{ETo} / \mathrm{RC})$, etc., can be plotted using a spider-plot presentation. The fluorescence transients and the spider presentation are thoroughly described by Strasser et al. (34). On a sample basis (per CS), the light absorption (ABS)/CSo (corresponding to the same amount of Chl) remained constant from 0 to $6 \mathrm{mM} \mathrm{PM- \alpha -CD}$, whereas the photon trapping (TRo)/CSo and electron transfers (ETo/CSo) decreased, in particular at 4 and $6 \mathrm{mM} \mathrm{PM-} \alpha-\mathrm{CD}$. However, the specific trapping (TRo/RC) and, to a lesser extent, electron transfers $(\mathrm{ETo} / \mathrm{RC})$ per reaction center were not affected up to about $6 \mathrm{mM}$. In contrast, the light absorption per active reaction center (ABS/RC) increased, especially from 0 to $4 \mathrm{mM}$ PM- $\alpha$-CD. In addition, the density of active reaction centers (RC/CSo) decreased steadily upon PM- $\alpha-\mathrm{CD}$ addition, whereas the overall quantum yield phi (Po) decreased only in the presence of the highest levels (4 to $6 \mathrm{mM}$ ) of CD.

\section{DISCUSSION}

The results of this investigation show that $\mathrm{CD}$, in particular their permethylated derivative, $\mathrm{PM}-\alpha-\mathrm{CD}$, offer interesting properties for removing glycerolipids from the TM. When comparing the lipid removal capacity of the different $\mathrm{CD}$ and derivatives (Table 1), we chose to investigate further the properties of PM- $\alpha-C D$ for the following reasons: (i) This compound removed 50 to $70 \mathrm{~mol} \%$ of anionic lipids (PG and SQDG) from the membrane (Fig. 2); (ii) at a concentration of $5 \mathrm{mM}$, it had almost no effect on galactolipids (Fig. 2); (iii) compared with $\alpha-C D$, the permethylated derivative displays a greater cavity opening of the larger ring edge of the molecule, which might enhance its capacity for engulfing lipid molecules (35); (iv) compared with all $\beta$-CD and its derivatives, the $\alpha-C D$ and PM- $\alpha-C D$ have the advantage of displaying a greater solubility in water (27); and (v) in contrast to detergent and enzymatic treatments, PM- $\alpha-C D$, up to a concentration of $6 \mathrm{mM}$, had no deleterious effect on the TM (Fig. 4), thereby avoiding special protective precautions, e.g., using high concentrations of BSA to remove FFA resulting from lipid digestion (20). All these properties make PM- $\alpha-C D$ a suitable compound for studying lipid composition-function relationships in TM.

The first set of results shows that, up to a concentration of 4 to $5 \mathrm{mM}$, PM- $\alpha$-CD displayed a marked selectivity for the removal of anionic lipids compared to galactolipids. Within this range of concentrations (Fig. 2), the structural integrity of the membrane was preserved (Fig. 4), and neither PSII nor PSI activity was affected (Table 2). For instance, 50\% removal of both SQDG and PG did not impair PSII and PSI electron flow activities. This surprising finding can be explained in view of earlier results concerning the effect of enzymatic digestion of membrane lipids by various phospholipase treatments $(7,20)$. As is well established, the TM is constituted of two monolayers that display an asymmetric distribution of their lipids (2). For instance, the outer monolayer contains $70 \mathrm{~mol} \% \mathrm{PG}$ and the inner one, $30 \mathrm{~mol} \%$. On the other hand, the enzymatic digestion of all the PG located in the outer monolayer did not affect PSII and PSI activities, even after the removal of the products (e.g., FA) resulting from the enzymatic digestion. In contrast, the removal of a small amount of PG in the inner monolayer drastically obliterates the activity of PSII (7).

Altogether, these experiments strongly suggest that the PG molecules that were removed by PM- $\alpha-C D$ originated from the outer monolayer only, since PSII and PSI activities were not altered. At a higher concentration of PM- $\alpha-\mathrm{CD}(9.6 \mathrm{mM})$, half of both MGDG and DGDG molecules were removed from the TM by this treatment (Fig. 2). At this high concentration of PM- $\alpha-C D$, the osmotic response was almost completely abolished, indicating that the structure of the TM was greatly impaired. Interestingly, in vivo, similar results were found in an Arabidopsis mutant characterized by a defective MGDG synthase gene (MGD1). The amount of MGDG in this mutant is reduced by $42 \%$ compared with the wild type. The MGDG deficiency of the mgd1 mutant is correlated with striking defects in chloroplast ultrastructure (36). A similar observation was made in an Arabidopsis mutant dgd1 where DGDG lipid content undergoes a $90 \%$ reduction compared to the wild type (37).

In contrast to electron flow activity, Chl fluorescence was the most sensitive photosynthetic parameter to be affected by a treatment of thylakoids with PM- $\alpha-C D$. Indeed, the ratio F695/F740 decreased from 1.45 in the control to 0.4 in the presence of $9.6 \mathrm{mM}$ of PM- $\alpha-C D$. Interestingly, the greatest decrease in the ratio occurred only when anionic lipids were removed by the $C D$, i.e., at a concentration of $4 \mathrm{mM} \mathrm{PM-} \alpha-\mathrm{CD}$, about $50 \%$ of SQDG and $50 \%$ of PG were removed, whereas the pools of MGDG and DGDG were essentially intact. The decrease in the ratio F695/F740 was mainly due to the diminution of the fluorescence in PSII, thus suggesting that the removal of PG alters the organization of the light-harvesting Chl $a / b$ protein complex (LHCII) (see Ref. 38 for a discussion of relations between the trimerization of LHCII, grana stacking, and light energy distribution in photosynthetic membranes).

These results also argue in favor of the existence of bulk and specific lipid molecules in the TM. The characteristics of these two types of molecules have recently been discussed $(9,10)$. Thus, most of the PG and SQDG molecules that are removed from the outer monolayer at low concentrations of PM- $\alpha-C D$ (up to 4-5 mM) can be considered as being essentially bulk molecules since PSII and PSI electron flow activities are not impaired. However, one cannot exclude that certain specific anionic lipids, probably PG molecules, are also removed, thus altering the molecular organization of LHCII and its related fluorescence. At higher concentrations of PM$\alpha-\mathrm{CD}(>4 \mathrm{mM})$, the removal of only a few specific molecules of DGDG may change the conformation of LHCII proteins. Indeed, PG molecules have been reported to be essential in 
the formation of LHCII trimers and its two- and three-dimensional crystallization. Furthermore, DGDG was found to bind to peripheral sites of LHCII (four molecules of DGDG per polypeptide) and to be crucial for the three-dimensional crystallization. Thus, specific PG and DGDG are both important molecules for the maintenance of the structure and function of LHCII (38-41). According to the spider-plot representation, the electron transfer (beyond $\mathrm{Q}_{\mathrm{A}}^{-}$) decreased per cross section $(\mathrm{ETo} / \mathrm{CSo})$ and remained constant per active reaction center $(\mathrm{ETo} / \mathrm{RC})$ in PM- $\alpha$-CD-treated TM. Thus, it is concluded that the fraction of reaction centers $(\mathrm{RC})$ inactivated (inactive RC/active RC + inactive RC) by the removal of lipids increased while the active $\mathrm{RC}$ remained intact but decreased in number. A few authors $(14,15)$ have hypothesized how reaction centers can be inactivated. Certain bound SQDG and DGDG molecules are localized as prosthetic groups at the surface of the native $\mathrm{D}_{1} / \mathrm{D}_{2}$ heterodimer, holding the dimer together, whereas PG can be considered to be essential for the orientation and stabilization of the $\mathrm{D}_{1}$-protein. Ionic interactions and van der Waal's forces in hydrophobic pockets or clefts might be the principal binding system between $\mathrm{D}_{1}$ and PG. For instance, after PLA $\mathrm{A}_{2}$ treatment $(14,15)$ or after removal of PG by PM- $\alpha-C D$, the cleft could be closed and the function of the lipoprotein complex $\left(\mathrm{D}_{1}\left(\mathrm{Q}_{\mathrm{B}}\right)\right.$ and $\mathrm{D}_{2}\left(\mathrm{Q}_{\mathrm{A}}\right)$ in the reaction center) obliterated. These changes might correspond to an increase of the fraction of inactivated reaction centers.

The capacity of CD to engulf lipids is the result of several properties of not only the host (i.e., CD) but also the guest (i.e., lipids) molecules. Concerning the host molecule, the number of glucopyranose units, and the diameter of the cavity (26), the nature of the chemical groups (hydroxyl and methoxy groups) at the edges of the cavity $(27,35)$, the polarity of each cyclic unit molecule, and the distribution of the charges, etc., are relevant factors in understanding the ability of $\mathrm{CD}$ to form water-soluble guest-CD inclusion complexes (27). On the other hand, the chemical properties of the guest molecules, such as the global charges of lipid molecules, the electrostatic properties of the lipid headgroups, and the nature of the acyl chains of the lipids (length and degree of unsaturation), are also determinative for generating this engulfing process (24). In our case, the proportion of removable lipids originating from a membrane should also depend on the specific characteristics of the membrane where lipids are inserted, namely, the transmembrane distribution of lipids, the packing pressure of the membrane, and the accessibility of lipid molecules to $\mathrm{CD}$. Although this investigation was not aimed at studying the host-guest interactions, our results may reveal information about this process.

First, let us consider the host characteristics: It has been reported that the permethylation of $\mathrm{CD}$ enlarges the whole cavity of the molecule (35). The methyl groups introduced into the $\mathrm{O}_{3}$ position extend the $\mathrm{O}_{2}-\mathrm{O}_{3}$ side of the cavity and make narrower the $\mathrm{O}_{6}$ side (27), thus favoring the formation of inclusion complexes. Our results are in agreement with this observation (Table 1). The removal of all four classes of lipids was increased in the presence of PM- $\alpha-C D$ compared with
$\alpha-C D$ as well as in the presence of 2,6 DM- $\beta-C D$ compared with the $\beta-\mathrm{CD}$. When considering the guest molecule, the presence of negative charge in the molecule (SQDG and PG) allowed a better interaction with the host molecule (Table 1). Interestingly, Debouzy et al. (24) used liposomes (multilamellar vesicles) to study the overall sensitivity of different phospholipids for their interactions with $\alpha-C D$. They found that among the most reactive phospholipids appeared PI and PS, which are both anionic lipids. However, PE, but not sphingomyelin and $\mathrm{PC}$, which are characterized by a zero global charge, is also reactive toward $\alpha-C D$. Obviously, the global charge of lipids appears not to be the only criterion influencing the formation of the host-guest complex. On the other hand, the nature of the acyl chains of the anionic lipids is apparently not decisive for the recognition of the guest by the host (Fig. 3). An intriguing question concerns the high concentration of PM- $\alpha-\mathrm{CD}$ (up to $5 \mathrm{mM}$ ) necessary to initiate the removal of galactolipids from the membrane. As discussed by Bekers et al. (27), it may be possible that more than one molecule of the host is necessary to find complete accommodation within the inclusion complex. In addition to that, the lack of global charges in galactolipids may require higher concentrations of PM- $\alpha-C D$.

\section{ACKNOWLEDGMENTS}

This research was supported in part by the Swiss National Science Foundation (grants number 31,336,93,92 and 31,432,97,95). The authors thank very much Dr. Saturnin Claude for his generous gift of PM- $\alpha-C D$ and Dr. Reto Strasser (University of Geneva) for making his laboratory available for fluorescence transient analyses and for helping to interpret the fluorescence data. This work is part of a doctoral program that was carried out by Sylvie Duchêne in the Laboratoire de Physiologie végétale, Université de Neuchâtel, Switzerland.

\section{REFERENCES}

1. Dubacq, J.P., and Trémolières, A. (1983) Occurrence and Function of Phosphatidylglycerol Containing $\Delta^{3}$-trans-Hexadecenoic Acid in Photosynthetic Lamellae, Physiol. Vég. 21, 293-312.

2. Siegenthaler, P.A. (1998) Molecular Organization of Acyl Lipids in Photosynthetic Membranes of Higher Plants, in Lipids in Photosynthesis: Structure, Function and Genetics (Siegenthaler, P.A., and Murata, N., eds.), Vol. 6, pp. 119-144, Kluwer Academic Publishers, Dordrecht.

3. Nishihara, M., Yokota, K., and Kito, M. (1980) Lipid Molecular Species Composition of Thylakoid Membranes, Biochim. Biophys. Acta 617, 12-19.

4. Xu, Y.N., and Siegenthaler, P.A. (1996) Phosphatidylglycerol Molecular Species of Photosynthetic Membranes Analyzed by High-Performance Liquid Chromatography: Theoretical Considerations, Lipids 31, 223-229.

5. Rawyler, A., and Siegenthaler, P.A. (1985) Transversal Localization of Monogalactosyldiacylglycerol in Spinach Thylakoid Membrane, Biochim. Biophys. Acta 815, 287-298.

6. Rawyler, A., Unitt, M.D., Giroud, C., Davies, H., Mayor, J.P., Harwood, J.L., and Siegenthaler, P.A. (1987) The Transmembrane Distribution of Galactolipids in Chloroplast Thylakoids Is Universal in a Wide Variety of Temperate Climate Plants, Photosynth. Res. 11, 3-13.

7. Siegenthaler, P.A., Rawyler, A., and Smutny, J. (1989) The Phospholipid Population Which Sustains the Uncoupled Non-cyclic 
Electron Flow Activity Is Localized in the Inner Monolayer of the Thylakoid Membrane, Biochim. Biophys. Acta 975, 104-111.

8. Duchêne, S., Smutny, J., and Siegenthaler, P.A. (2000) The Topology of Phosphatidylglycerol Populations Is Essential for Sustaining Photosynthetic Electron Flow Activities in Thylakoid Membranes, Biochim. Biophys. Acta 1463, 115-120.

9. Duchêne, S., and Siegenthaler, P.A. (2000) Do Glycerolipids Display Lateral Heterogeneity in the Thylakoid Membrane? Lipids 35, 739-744.

10. Siegenthaler, P.A., and Trémolières, A. (1998) Role of Acyl Lipids in the Function of Higher Plants Photosynthetic Membranes, in Lipids in Photosynthesis: Structure, Function and Genetics (Siegenthaler, P.A., and Murata, N., eds.), Vol. 6, pp. 145-173, Kluwer Academic Publishers, Dordrecht.

11. Rawyler, A., and Siegenthaler, P.A. (1989) Change in the Molecular Organization of Monogalactosyldiacylglycerol Between Resting and Functioning Thylakoid Membranes. Involvement of the $\mathrm{CF}_{0} / \mathrm{CF}_{1}$-ATP Synthetase, Biochim. Biophys. Acta 975 , 283-292.

12. Siegenthaler, P.A., Sutter, J., and Rawyler, A. (1988) The Transmembrane Distribution of Galactolipids in Spinach Thylakoid Inside-Out Vesicles Is Opposite to That Found in Intact Thylakoids, FEBS Lett. 228, 94-98.

13. Radunz, A. (1981) Application of Antibodies in the Analysis of Structural Configuration of Thylakoid Membranes, Ber. Deutsch. Bot. Ges. 94, 477-489.

14. Voss, R., Radunz, A., and Schmid, G.H. (1992) Binding of Lipids onto Polypeptides of the Thylakoid Membrane. I. Galactolipids and Sulpholipid as Prosthetic Groups of Core Peptides of the Photosystem II Complex, Z. Naturforsch. 47c, 406-415.

15. Kruse, O., and Schmid, G.H. (1995) The Role of Phosphatidylglycerol as a Functional Effector and Membrane Anchor of the $\mathrm{D}_{1}$-Core Peptide from Photosystem-II Particles of the Cyanobacterium Oscillatoria chalybea, Z. Naturforsch. 50c, 380-390.

16. Murata, N., Higashi, S.I., and Fujimura, K. (1990) Glycerolipids in Various Preparations of Photosystem II from Spinach Chloroplasts, Biochim. Biophys. Acta 1019, 261-268.

17. Trémolières, A., Dainese, P., and Bassi, R. (1994) Heterogenous Lipid Distribution among Chlorophyll-Binding Proteins of Photosystem II in Maize Mesophyll Chloroplasts, Eur. J. Biochem. 221, 721-773.

18. Vijayan, P., Routaboul, J.M., and Browse, J. (1998) A Genetic Approach to Investigating Membrane Lipid Structure and Photosynthetic Function, in Lipids in Photosynthesis: Structure, Function, and Genetics (Siegenthaler, P.A., and Murata, N., eds.), Vol. 6, pp. 263-285, Kluwer Academic Publishers, Dordrecht.

19. Henry, L.E.A., Mikkelsen, J.D., and Møller, B.L. (1983) Pigment and Acyl Lipid Composition of Photosystem I and II Vesicles and of Photosynthetic Mutants in Barley, Carlsberg Res. Commun. 48, 131-148.

20. Siegenthaler, P.A., Smutny, J., and Rawyler, A. (1987) Involvement of Distinct Populations of Phosphatidylglycerol and Phosphatidylcholine Molecules in Photosynthetic Electron-Flow Activities, Biochim. Biophys. Acta 891, 85-93.

21. Murphy, D.J., and Woodrow, I.E. (1983) Lateral Heterogeneity in the Distribution of Thylakoid Membrane Lipid and Protein Components and Its Implications for the Molecular Organization of Photosynthetic Membranes, Biochim. Biophys. Acta 725, 104-112.

22. Sundby, C., and Larsson, C. (1985) Transbilayer Organization of the Thylakoid Galactolipids, Biochim. Biophys. Acta 813, 61-67.

23. Rawyler, A., and Siegenthaler, P.A. (1996) Cyclodextrins: A New Tool for the Controlled Lipid Depletion of Thylakoid Membranes, Biochim. Biophys. Acta 1278, 89-97.

24. Debouzy, J.C., Fauvelle, F., Crouzy, S., Girault, L., Chapron, Y., Göschl, M., and Gadelle, A. (1998) Mechanism of $\alpha$-Cyclodextrin Induced Hemolysis. 2. A Study of the Factors Controlling the Association with Serine-, Ethanolamine-, and
Choline-Phospholipids, J. Pharm. Sci. 87, 59-66.

25. Szejtli, J. (1988) Topics in Inclusion Science, in Cyclodextrin Technology (Davies, J.E.D., ed.), pp. 1-306, Kluwer Academic Publishers, Dordrecht.

26. Szejtli, J. (1990) The Cyclodextrins and Their Applications in Biotechnology, Carbohydrate Polymers 12, 375-392.

27. Bekers, O., Uijendaal, E.V., Beijnen, J.H., Bult, A., and Underberg, W.J.M. (1991) Cyclodextrins in the Pharmaceutical Field, Drug Dev. Indus. Pharm. 17, 1503-1549.

28. Duchêne, S., Strasser, R.J., Srivastava, A., and Siegenthaler, P.A. (1998) Cyclodextrins as a Tool for Studying the Role of Glycerolipids in Spinach Thylakoid Membranes, in Proceedings of the 10th International Congress on Photosynthesis, Budapest, Hungary (Garab, G., ed.), Kluwer Academic Publishers, Dordrecht.

29. Duchêne, S. (1998) Les Glycérolipides: Organisation Spatiale et Fonctions dans les Membranes Photosynthétiques de l'Epinard. Etude à l'Aide de Vésicules Inversées et de Cyclodextrines, Ph.D. thesis, University of Neuchâtel, pp. 1-118.

30. Xu, Y.N., and Siegenthaler, P.A. (1996) Effect of Non-Chilling Temperature and Light Intensity During Growth of Squash Cotyledons on the Composition of Thylakoid Membrane Lipids and Fatty Acids, Plant Cell Physiol. 37, 471-479.

31. Bruinsma, J. (1961) A Comment on the Spectrophotometric Determination of Chlorophyll, Biochim. Biophys. Acta 52, 576-578.

32. Srivastava, A., and Strasser, R.J. (1996) Stress and Stress Management of Land Plants During a Regular Day, J. Plant Physiol. $148,445-455$.

33. Nobel, P.S. (1970) Osmotic Responses of Chloroplasts, in Plant Cell Physiology, A Physicochemical Approach, pp. 56-57, W.H. Freeman and Company, San Francisco.

34. Strasser, R.J., Srivastava, A., and Tsimilli-Michael, M. (2000) The Fluorescence Transient As a Tool to Characterize and Screen Photosynthetic Samples, in Probing Photosynthesis: Mechanisms, Regulation, and Adaptation (Yunus, M., Pathre, U., and Mohanty, P., eds.), pp. 445-483, Taylor \& Francis, London and New York.

35. Reinhardt, R., Richter, M., and Mager, P.P.(1996) Investigation of the Conformational Behaviour of Permethylated Cyclodextrins by Molecular Modelling, Carb. Res. 291, 1-9.

36. Jarvis, P., Dörmann, P., Peto, C.A., Lutes, J., Benning, C., and Chory, J. (2000) Galactolipid Deficiency and Abnormal Chloroplast Development in the Arabidopsis MGD Synthase 1 Mutant, Proc. Natl. Acad. Sci. USA 97, 8175-8179.

37. Dörmann, P., Balbo, I., and Benning, C. (1999) Arabidopsis Galactolipid Biosynthesis and Lipid Trafficking Mediated by DGD1, Science 284, 2181-2184.

38. Trémolières, A., and Siegenthaler, P.A. (1998) Reconstitution of Photosynthetic Structures and Activities with Lipids, in Lipids in Photosynthesis: Structure, Function, and Genetics (Siegenthaler, P.A., and Murata, N., eds.), Vol. 6, pp. 175-189, Kluwer Academic Publishers, Dordrecht.

39. Nussberger, S., Dörr, K., Wang, D.N., and Kühlbrandt, W. (1999) Lipid-Protein Interaction in Crystals of Plant LightHarvesting Complex, J. Mol. Biol. 234, 347-356.

40. Kühlbrandt, W., Wang, D.N., and Fujiyoshi, Y. (1994) Atomic Model of Plant Light-Harvesting Complex by Electron Crystallography, Nature 367, 614-621.

41. Peterman, E.J.G., Hobe, S., Calkoen, F., van Grandelle, R., Paulsen, H., and van Ameronges, H. (1996) Low-Temperature Spectroscopy of Monomeric and Trimeric Forms of Reconstituted Light-Harvesting Chlorophyll $a / b$ Complex, Biochim. Biophys. Acta 1273, 171-174.

[Received July 2, 2001, and in revised form November 2, 2001; revision accepted November 9, 2001] 